\title{
The Regularity of Science in Moonlight Sonata Instrument is used in The Design of Physics Learning
}

\author{
Sofa Maria \\ Physics Education Department, Faculty of Teacher Training and Education, Universitas Sebelas Maret \\ Surakarta, Indonesia \\ sofamaria10@gmail.com
}

\begin{abstract}
The main purpose of edutainment is to increase enthusiasm for learning. However, the implementation of physics learning still lacks emphasis on the learning process that meaningful and relevant, especially in Indonesia. This study was purposed to make the design of physics learning by integrating science with music, that is applying usage Moonlight Sonata by Ludwig Van Beethoven as a physical learning instrument in sound wave material. The results of the design were learning guide that contains the regularity of science in Moonlight Sonata instrument, and the experiment of making a simple musical instrument using these scientific regularities. The principle of learning design was showed the regularity of the sound waves on the Moonlight Sonata instrument along with explanations using basic physics concepts about the sound wave. Based on the study results concluded that the learning guide can be used as an instrument that delivers to the design of physics learning that meaningful and relevant.
\end{abstract}

Keywords-regularity of science, Moonlight Sonata, physics, sound waves, learning design

\section{INTRODUCTION}

In this era, the rapid development of science requires us to create an educational culture that remains relevant, so that it requires innovation in learning strategies. However, the implementation of physics learning still lacks the development of learning strategies, especially in Indonesia. This seizes the attention of the forming learning culture that meaningful and relevant. Meaningful and relevant learning can be achieved through science learning carried out by integrating learning material with reality in everyday life. Therefore, it is necessary to develop strategies in the delivery of knowledge so that students are able to have creativity, empirical experience, and complex thinking power. One of the development strategies namely by giving a discourse on the integration of science and art based on the application of physics in music.

The coached teacher was reported greater confidence integrating the arts, produced higher-quality work samples, taught more reading concepts with arts integration, implemented more arts standards, and used arts integration more frequently than did the institute-only teachers or the control group teachers. Institute-only teachers demonstrated greater confidence in, and used arts integration more frequently, than did the comparison group. However, they did not reach the same levels as the coached teachers and were more likely to report time constraints and other roadblocks to successful implementation.

In this fact, students still lack the experience to apply the sound waves material concept by physics learning in the classroom. It is necessary to use learning media that are relevant to the material; one of them is the media of musical instruments in the form of Moonlight Sonata by Ludwig Van Beethoven as an instrument in developing physics learning strategies.

The proposed of this research were explained the regularity of science in Moonlight Sonata as a musical instrument. Mathematical exposure of regularity was using the basic concepts of sound waves, investigating the regularity of the frequency of waves that form a harmony. A musical composition of harmony can be formed by these regularities. The results of the explanation about the regularity of science in the Moonlight Sonata will be used as a theoretical basis in the learning guide. The learning guide contains the task to do experiments by making simple musical instruments in the form of sheet music by applying the regularity of science in creating musical harmony.

It is hoped that the Moonlight Sonata can be an instrument of development in physics learning. This learning guide is made by the aimed of becoming a discourse in learning design that takes meaning and relevance. However, it is hoped that there will be further research on the success and effectiveness of applying this learning design in the classroom.

\section{THE REGULARITY OF SCIENCE IN MUSICAL INSTRUMENT}

\subsection{The Regularity of Science in Music}

The regularity of science can be seen from a pattern. Patterns were formed by repetition, symmetry, pairing, form, etc. Investigating the regularity of musical greetings in music can be a form of exploring physical concepts with naturally immeasurable ideas (feelings).

Naturally, many elements that influence each other are the opposite thing that becomes a unity, as the opposite thing appears in pairs, together to maintain each other's existence. No exception with music, we can say that harmony can be created from a combination of sounds that resonate and sound dissonance. Where consonance has regularity in the composition of the wave and dissonance 
has no regularity in the composition of the wave. The unity of consonance and dissonance sounds may be the reason for the unmeasurable mathematical harmony which has the effect of making music sound beautiful and epic. It is a natural unity of opposing elements.

\subsection{Science and Music}

Music art is science and art itself. Music is a sound composition that forms a harmony of tones and has a rhythm. In the creation of music, there are musical instruments that support it. Musical instruments are instruments that are made or modified to create music. One of the instruments of musical art or called musical instruments is sheet music, which is written music.

Certainly, there is the presence of rhythm in the sounds we call music. At a more sophisticated level, there is the presence of those who combine with other orders and "pleasing" ways. Noise is often associated with very loud and grating sounds that don't sound together or are somehow "unpleasant" [1]. However, in everyday life, people have different perceptions of music. Subjectively, somehow there are people who think that something is called music and some consider it just to be noisy. In this paper, we will equate perception of music, objectively in terms of the regularity of science.

Science and art have similarities which are based on processes in the form of power development, creativity, imagination, and synthesis ability. Modelling of natural laws and works of art (such as paintings, songs, music, etc.) are the result of encouragement by scientists and artists to represent nature in accordance with their perceptions. In this paper, the discussion is integrating music and musical instruments with physics about waves and sounds, investigating the regularity of waves by looking at their frequency in musical instruments to see the harmony structure of the tones that make up consonance.

Beethoven's work with his ability to give emotional elements and immeasurable creativity to mathematical provisions, make things called Hector Berlioz as "one of the poems that human language cannot measure its quality". (https:/youtu.be/zAxT0mRGuoY). These youtube showed the power of the melody of music can influence, create, and convey feelings. So that music can be communication that goes beyond language, which can touch someone emotionally. So by using music in science learning, we can provide experiences to students physically and emotionally.

\subsection{Sound Waves}

Waves are one of the most important in the modern world, like in the music industry. Waves are vibrations that propagate. Waves are transfers of energy without the transfer of material. One type of wave is a mechanical wave that can exist only in media material and is governed by Newton's laws. An example of mechanical waves is a sound wave; where transverse sound waves need the particles of the medium (such as water and rock) oscillate perpendicular to the wave's direction of travel that is longitudinal waves.

A sinusoidal wave moving in the positive direction of an $\mathrm{X}$-axis has the mathematical form

\begin{tabular}{|c|c|}
\hline$y(x, t)=y_{m} \sin (k x-\omega t)$ & (1) \\
\hline
\end{tabular}

Where $y_{m}$ is the amplitude (magnitude of the maximum displacement) of the wave, $k$ is the angular wavenumber,

$\omega$ is the angular frequency, and $k x-\omega t$ is the phase. The wavelength $\lambda$ is related to $k$ by

$$
y(x, 0)=y_{m} \sin k x \quad(t=0)
$$

$$
\begin{aligned}
& \text { at } x=x_{1} \text { and } x=x_{1}+\lambda \\
& \begin{aligned}
y_{m} \sin k x_{1} & =y_{m} \sin k\left(x_{1}+\lambda\right) \\
& =y_{m} \sin \left(k x_{1}+k \lambda\right)
\end{aligned}
\end{aligned}
$$

A sine function begins to repeat itself when its angle (or argument) is increased by $2 \pi \mathrm{rad}$, so in Equation 3 we must have $k \lambda=2 \pi$, or

$$
k=\frac{2 \pi}{\lambda} \text { (angular wavenumber) }
$$

\subsection{Frequency}

Frequency is the number of vibrations that occur in one second, with a unit of $1 / \mathrm{s}$ called Hertz $(\mathrm{Hz})$. The higher frequency value, the more vibrations/waves produced, so the higher the frequency produced by the sound source, the higher the sound produced.

$$
f=\frac{n}{t}
$$

\section{Information:}

$f$ : frequency (Hertz)

$n$ : number of vibrations/waves

$t$ : time (second)

So, the frequency is a number of oscillations per unit time.

\subsection{Period}

A period is a time needed to do one vibration/wave.

$$
T=\frac{t}{n}
$$

Information:

$T$ : period (second)

$t$ : time (second)

$n$ : number of vibrations/waves, with $n=1$

So, the period of oscillation $\mathrm{T}$ of a wave to be the time any string element takes to move through one full oscillation. 


\subsection{Angular Frequency}

The displacement $y$ of Equation (3.1) versus time $t$ at a certain position along the string, taken to be $x=0$. The string position moves up and down in simple harmonic motion.

The equation of the simple harmonic motion:

\begin{tabular}{|cc|c|}
$y(0, t)=y_{m} \sin (-\omega t)$ &,$(x=0)$ & (7) \\
& $=-y_{m} \sin \omega t$ &
\end{tabular}

It can be true only if $\omega T=2 \pi$, or if

$$
\omega=\frac{2 \pi}{T}
$$

\section{Information:}

$\omega:$ angular frequency (radians)

$T$ : period (second)

$2 \pi$ : one round (radians)

So, the angular frequency of the wave; it's the unit is the radian per second.

The period $T$ and frequency $f$ of the wave is related to $v$ by

$$
\frac{\omega}{2 \pi}=f=\frac{1}{T}
$$

The wave speed $v$ (the speed of the wave along the string) is related to these other parameters by

$$
v=\frac{\omega}{k}=\frac{\lambda}{T}=\lambda f
$$

Any function of the form

$$
y(x, t)=h(k x \pm \omega t)
$$

This formula can represent a travelling wave with a wave speed as given above and a wave shape given by the mathematical form of $h$. The plus sign denotes a wave travelling in the negative direction of the $\mathrm{x}$-axis, and the minus signs a wave travelling in the positive direction. [2].

\section{RESULT AND DISCUSSION}

The results of the design were learning guide form that contains the regularity of science in Moonlight Sonata instrument, and the experiment of making a simple musical instrument using these scientific regularities. Experiment using the students rules and looks the example before. The examples have shown an experiment that uses the regularity of science in a musical instrument.

\section{A. Learning Guide}

\section{Basic Theory}

a. Sheet Music and The Wave Pattern of Musical Instrument in Moonlight Sonata

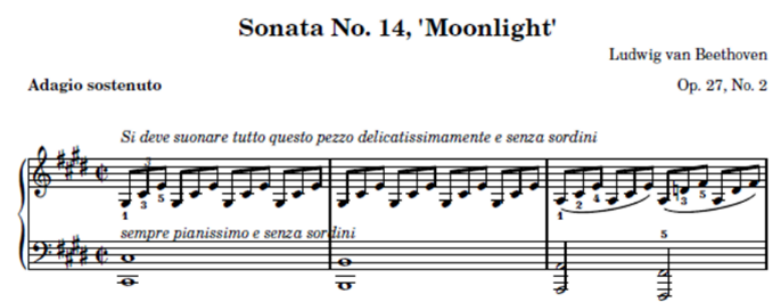

Fig. 1. Sheet music of the Moonlight Sonata

With the following rules:

\begin{tabular}{|l|l|l|l|l|l|l|l|l|l|l|l|l|l|l|}
\hline 1 & 2 & & 3 & 4 & 5 & 6 & & 7 & 8 & 9 & & 10 & 11 & 12 \\
\hline & & & & & & & & & & & & & \\
\hline $\mathrm{D}$ & & \multicolumn{3}{|c|}{$\mathrm{F}^{*}$} & $\mathrm{~A}$ & & & & & & & \\
\hline \multicolumn{2}{|l|}{ terts } & & & & & & & & & \\
\hline
\end{tabular}

Fig. 2. The rules on the tuts of Piano

Information:

Triad: $\mathrm{D}-\mathrm{F}^{\#}-\mathrm{A}(1-3-5)$

A sinusoidal wave moving in the positive direction of an $x$ axis has the mathematical form.

$$
y(x, t)=y_{m} \sin (k x-\omega t)
$$

The angular frequency has the mathematical form

$$
\begin{array}{rlr}
y(0, t) & =y_{m} \sin (-\omega t) \quad,(x=0) \\
& =-y_{m} \sin \omega t &
\end{array}
$$

As we have known that the period $T$ and frequency $f$ of the wave is related to $v$ by

$$
\frac{\omega}{2 \pi}=f=\frac{1}{T}
$$

Then we have:

$$
y(t)=\sin (2 \pi f t)
$$

Information:

$f:$ frequency (Hertz)

$t$ : time(second)

Pay attention to triplet notes, and we have:

$$
\begin{gathered}
\text { - } \mathrm{A}_{4} \quad f=440 \mathrm{~Hz} \\
y(t)=\sin (2 \pi 440 t) \\
\text { - } \mathrm{F}_{4}^{\#} \quad f=370 \mathrm{~Hz} \\
y(t)=\sin (2 \pi 370 t) \\
\text { - } \mathrm{D}_{4} \quad f=294 \mathrm{~Hz} \\
y(t)=\sin (2 \pi 294 t) \\
\text { Triplet: } \mathrm{E}-\mathrm{G}^{\#}-\mathrm{B}(5-9-12)
\end{gathered}
$$

Why some note combinations sound better? Let's use middle $\mathrm{C}$ and the $\mathrm{G}$ just above it as an example: 


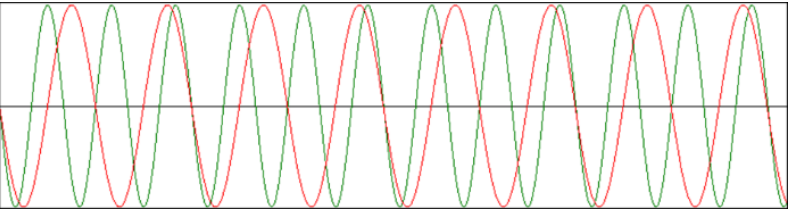

Fig. 3. The waveform of $\mathrm{C}$ and $\mathrm{G}$ notes

Now let's look at two notes that sound terrible together, C and $\mathrm{F}^{\#}$ :

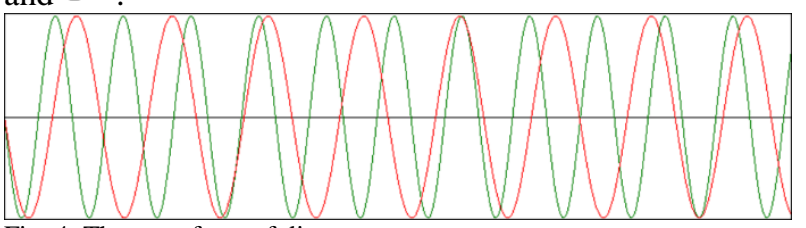

Fig. 4. The waveform of dissonance

Based on waveform analysis using Fourier analysis, the wave images formed from $\mathrm{C}$ and $\mathrm{G}$ simultaneously have the regularity that every 3 rd wave of the $\mathrm{G} 2$ nd wave of the $\mathrm{C}$ matches. This combination of notes forms harmony with the match between the two notes seen from the meeting point of the two waves that shows regularity, which is called consonance.

$\frac{392.0}{261.6}=1.4985$

The ratio corresponds to the ratio of the number of waves at the meeting point between wave $\mathrm{C}$ waves and $\mathrm{G}$ tone waves.

While Fourier analysis for waves formed from sounds of $\mathrm{C}$ note and $\mathrm{F}^{\#}$ note simultaneously has no regularity or is called dissonance.

From the frequency ratio, namely:

$$
\frac{370}{261.1}=1.4144 \text { or } \approx 1.5 \text { but never be } 1.5 \text {. }
$$

Example:

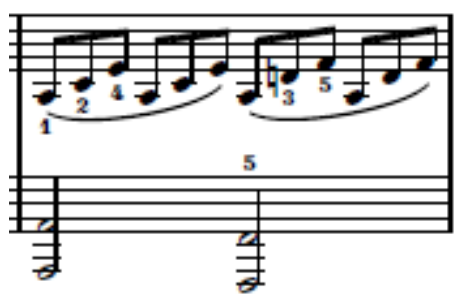

Fig. 5. Triad: $\mathrm{D}-\mathrm{F}^{\#}-\mathrm{A}(1-3-5)$

With a comparison of the frequencies of the three tones sequent around 1.125: 1.4: 1.66 predicted waves of all three tones will meet at comparison sequent wavelengths around 3.5: $3: 2.5$. This can be made possible by the formation of consonance which naturally forms three perfect ratios to $\mathrm{C}$ notes.

\section{b. Music}

There are chromatic scales, that the scales with a fixed voice. The frequency is called the chromatic scale with the same loudness. The interval of the octave with prime is divided into 12 equal voices, so they are each magnitude.

For example, middle $\mathrm{C}$ has a frequency of $262 \mathrm{~Hz}$ whereas ( $\mathrm{C}$ above middle $\mathrm{C}$ ) has twice that frequency, 524 Hz. That middle $\mathrm{C}$ is the $\mathrm{C}$ or "do" note at the middle of a piano keyboard. The following is equally tempered chromatic scale with only one octave is included:

\begin{tabular}{|l|c|c|}
\hline \multicolumn{3}{|c|}{ TABLE 1. Equally tempered chromatic scale. } \\
\hline Note & Name & Frequency (Hz) \\
\hline $\mathrm{C}$ & Do & 262 \\
\hline $\mathrm{C}^{\#}$ or $\mathrm{D}^{\mathrm{b}}$ & & 277 \\
\hline $\mathrm{D}$ & $\mathrm{Re}$ & 294 \\
\hline $\mathrm{D}_{\text {or }}^{\#}$ & & 311 \\
\hline $\mathrm{E}$ & $\mathrm{Mi}$ & 330 \\
\hline $\mathrm{F}$ & $\mathrm{Fa}$ & 349 \\
\hline $\mathrm{F}^{\#}$ or ${ }^{\mathrm{b}}$ & & 370 \\
\hline $\mathrm{G}$ & $\mathrm{Sol}$ & 392 \\
\hline $\mathrm{G}^{\#}$ or $\mathrm{A}$ & & 415 \\
\hline $\mathrm{A}$ & $\mathrm{La}$ & 440 \\
\hline $\mathrm{A}^{\#}$ or $\mathrm{B}$ & & 466 \\
\hline $\mathrm{B}$ & $\mathrm{Si}$ & 494 \\
\hline $\mathrm{C}^{\prime}$ & Do & 524 \\
\hline
\end{tabular}

\begin{tabular}{|c|c|c|c|c|}
\hline Note & $\begin{array}{c}\text { Perfect } \\
\text { Ratio to } \\
\text { C }\end{array}$ & $\begin{array}{c}\text { Actual } \\
\text { Ratio to } \\
\text { C }\end{array}$ & $\begin{array}{l}\text { Ratio } \\
\text { off by }\end{array}$ & $\begin{array}{c}\text { Frequency in } \\
\mathrm{Hz}\end{array}$ \\
\hline Middle C & & & & 261.6 \\
\hline $\mathrm{D}$ & $\begin{array}{ll}9 / 8 & \text { or } \\
1.125 & \end{array}$ & 1.1224 & 0.0026 & 293.7 \\
\hline $\mathrm{E}$ & $\begin{array}{ll}5 / 4 & \text { or } \\
1.25 & \end{array}$ & 1.2599 & 0.0099 & 329.6 \\
\hline $\mathrm{F}$ & $\begin{array}{l}4 / 3 \quad \text { or } \\
1.333 \ldots\end{array}$ & 1.3348 & 0.0015 & 349.2 \\
\hline G & $3 / 2$ or 1.5 & 1.4985 & 0.0017 & 392.0 \\
\hline A & $\begin{array}{l}5 / 3 \text { or } \\
1.666 \ldots\end{array}$ & 1.6818 & 0.0152 & 440.0 \\
\hline B & $\begin{array}{l}17 / 9 \text { or } \\
1.888 \ldots\end{array}$ & 1.8877 & 0.0003 & 493.9 \\
\hline
\end{tabular}

\section{Task}

Use the example below:

The $\mathrm{F}$ and $\mathrm{A}$ tones with the $\mathrm{F}$ to $\mathrm{C}$ ratio are $4 / 3$ and $\mathrm{A}$ to $\mathrm{C}$ were $5 / 3$, will meet at the point with the ratio $\mathrm{F}$ : $\mathrm{A}$ is $4: 5$. Musical instruments using predictions from the regularity above were to make a piece of simple sheet music. And these practices use equally tempered chromatic scales and the perfect ratio.

Rules:

Attach your work, that is sheet music and include its explanation about the regularity of science on your sheet music. 


\section{CONCLUSION}

Based on the study results concluded that the learning guide can be used as an instrument that delivers to the design of physics learning that meaningful and relevant. Using Moonlight Sonata can help to show the regularity of science in musical instrument simply to student know one of the examples how the implementation of sound waves concept in everyday life, that is for making written music. The explanation was using the basis sound waves concept and the theory of consonance. The learning guide is hoped to be the development of learning strategies.

\section{REFERENCES}

[1] A. L. Lynch, "Writing moonlight: an analysis of Beethoven's Piano Sonata Opus 27 No. 2 in C Sharp Minor,” vol. 6, pp. 30-32, 2007, in press.

[2] D. Halliday, R. Resnick, and J. Walker, "Fundamentals of Physics", 10th ed, vol. 16. John Wiley \& Sons, Inc.: the United States of America, pp. 444-448, 2014

[3] D. R Lapp, Fellow, "The Physics of Music and Musical Instruments, Wright Center for Science Education Tufts University": the United States of America, p. 3, 2003.

[4] D. C. Giancoli, "Physics Principles with Applications", 7th ed, vol. 12. Pearson Education, Inc.: the United States of America, pp. 335, 2014

[5] F. Capra, "The Tao of Physics: An Exploration of the Parallels Between Modern Physics and Eastern Mysticism", 4th ed, vol. 1. Jalasutra: Yogyakarta, p. 9, 2000.

[6] Ishafit, K. Anwar, and M. Toifur, "Musical Instrument Frequency scale measurement with a microcomputer-based laboratory system (Pengukuran Frekuensi Tangga Nada Instrumen Musik dengan Sistem Microcomputer Based Laboratory)," pp. 3-6, 2018, in press.

[7] P. A. Tipler, and G Mosca," Physics for Scientist and Engineers with Modern Physics", 6th ed, vol. 16. W. H. Freeman and Company: New York, pp. 552, 2008 